\title{
Erratum: The draft genome of the grass carp (Ctenopharyngodon idellus) provides insights into its evolution and vegetarian adaptation
}

Yaping Wang, Ying Lu, Yong Zhang, Zemin Ning, Yan Li, Qiang Zhao, Hengyun Lu, Rong Huang, Xiaoqin Xia, Qi Feng, Xufang Liang, Kunyan Liu, Lei Zhang, Tingting Lu, Tao Huang, Danlin Fan, Qijun Weng, Chuanrang Zhu, Yiqi Lu, Wenjun Li, Ziruo Wen, Congcong Zhou, Qilin Tian, Xiaojun Kang, Mijuan Shi, Wanting Zhang, Songhun Jang, Fukuan Du, Shan He, Lanjie Liao, Yongming Li, Bin Gui, Huihui He, Zhen Ning, Cheng Yang, Libo He, Lifei Luo, Rui Yang, Qiong Luo, Xiaochun Liu, Shuisheng Li, Wen Huang, Ling Xiao, Haoran Lin, Bin Han \& Zuoyan Zhu

Nat. Genet. 47, 625-631 (2015); published online 4 May 2015; corrected after print 15 July 2015

In the version of this article initially published, the affiliation for Songhun Jang was incorrectly listed as Kim Illinois Sung University, Pyongyang, North Korea. The correct affiliation is Kim Il Sung University, Pyongyang, North Korea. The error has been corrected in the HTML and PDF versions of the article.

\section{Corrigendum: Transcriptional regulator PRDM12 is essential for human pain perception}

Ya-Chun Chen, Michaela Auer-Grumbach, Shinya Matsukawa, Manuela Zitzelsberger, Andreas C Themistocleous, Tim M Strom, Chrysanthi Samara, Adrian W Moore, Lily Ting-Yin Cho, Gareth T Young, Caecilia Weiss, Maria Schabhüttl, Rolf Stucka, Annina B Schmid, Yesim Parman, Luitgard Graul-Neumann, Wolfram Heinritz, Eberhard Passarge, Rosemarie M Watson, Jens Michael Hertz, Ute Moog, Manuela Baumgartner, Enza Maria Valente, Diego Pereira, Carlos M Restrepo, Istvan Katona, Marina Dusl, Claudia Stendel, Thomas Wieland, Fay Stafford, Frank Reimann, Katja von Au, Christian Finke, Patrick J Willems, Michael S Nahorski, Samiha S Shaikh, Ofélia P Carvalho, Adeline K Nicholas, Gulshan Karbani, Maeve A McAleer, Maria Roberta Cilio, John C McHugh, Sinead M Murphy, Alan D Irvine, Uffe Birk Jensen, Reinhard Windhager, Joachim Weis, Carsten Bergmann, Bernd Rautenstrauss, Jonathan Baets, Peter De Jonghe, Mary M Reilly, Regina Kropatsch, Ingo Kurth, Roman Chrast, Tatsuo Michiue, David L H Bennett, C Geoffrey Woods \& Jan Senderek

Nat. Genet. 47, 803-808 (2015); published online 25 May 2015; corrected after print 8 July 2015

In the version of this article initially published, there was an error with the affiliations for author Roman Chrast. His correct affiliations are: Department of Medical Genetics, University of Lausanne, Lausanne, Switzerland; Department of Neuroscience, Karolinska Institutet, Stockholm, Sweden; and Department of Clinical Neuroscience, Karolinska Institutet, Stockholm, Sweden. The error has been corrected in the HTML and PDF versions of the article.

\section{Corrigendum: Statistical colocalization of genetic risk variants for related autoimmune diseases in the context of common controls}

Mary D Fortune, Hui Guo, Oliver Burren, Ellen Schofield, Neil M Walker, Maria Ban, Stephen J Sawcer, John Bowes, Jane Worthington, Anne Barton, Steve Eyre, John A Todd \& Chris Wallace

Nat. Genet. 47, 839-846 (2015); published online 8 June 2015; corrected after print 8 July 2015

In the version of this article initially published, the two panels in Figure 2 were presented in the incorrect order. The error has been corrected in the HTML and PDF versions of the article. 